Du texte à la scène : langages du théâtre

\title{
Pierre Étienne Heymann : à propos de la mise en scène de Macbeth par « La Rose des Vents » à Villeneuve d'Ascq
}

Pierre Étienne Heymann

\section{(2) OpenEdition \\ Journals}

Édition électronique

URL : http://journals.openedition.org/shakespeare/477

DOI : 10.4000/shakespeare.477

ISSN : 2271-6424

Éditeur

Société Française Shakespeare

Édition imprimée

Date de publication : 1 novembre 1983

Pagination : 247-250

Référence électronique

Pierre Étienne Heymann, « Pierre Étienne Heymann : à propos de la mise en scène de Macbeth par « La Rose des Vents » à Villeneuve d'Ascq », Actes des congrès de la Société française Shakespeare [En ligne], 4 | 1983, mis en ligne le 01 janvier 2007, consulté le 27 avril 2019. URL : http:// journals.openedition.org/shakespeare/477 ; DOI : 10.4000/shakespeare.477 
SOCIETE FRANÇAISE SHAKESPEARE

Actes du Congrès 1982

\section{DU TEXTE A LA SCENE : Langages du Théâtre}

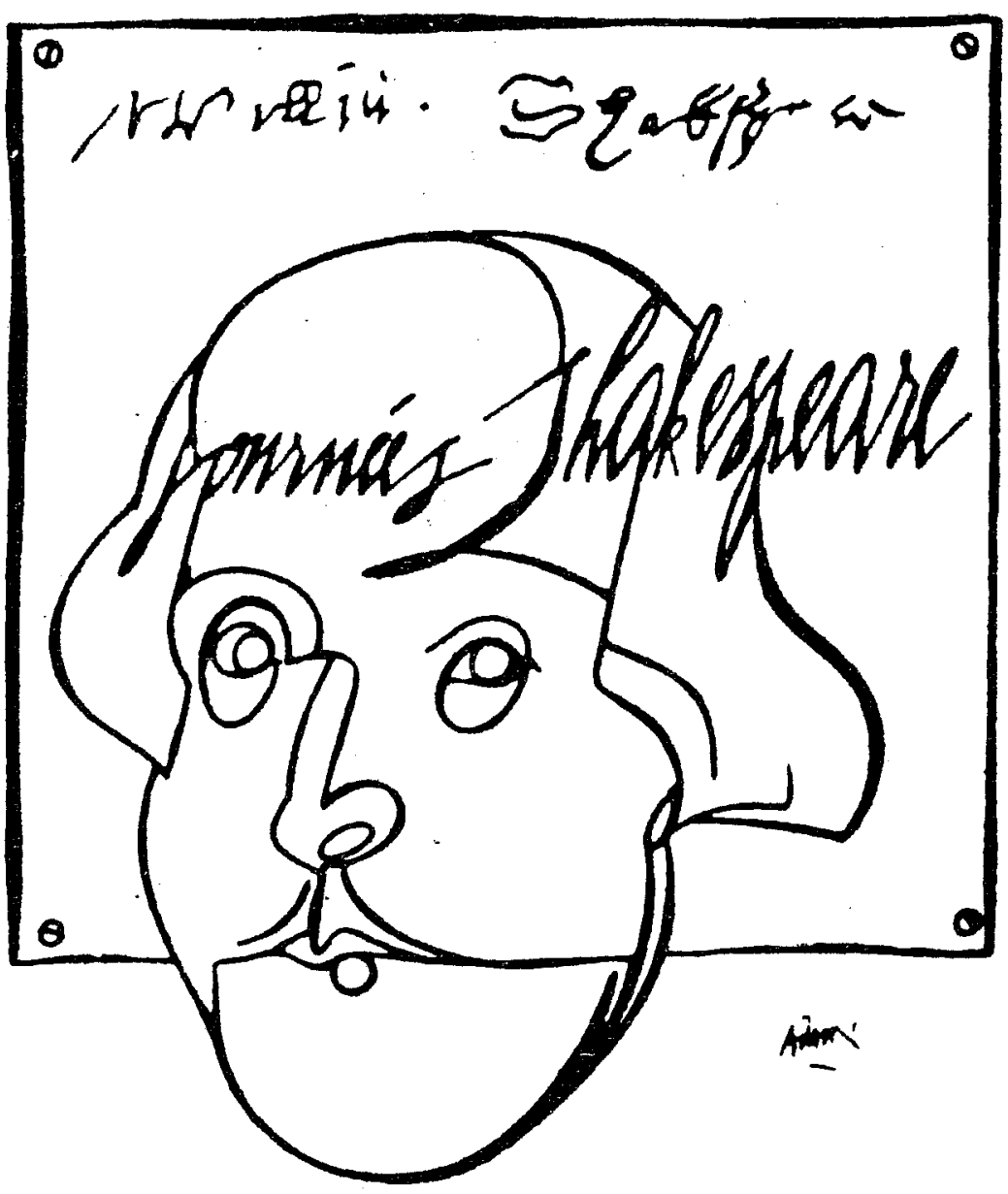

DiRecteur de la publication M.T. Jones - Davies

Publié avec le concours du Centre National de la Recherche Scientifique

JEAN TOUZOT Libraire - Editeur

38 , rue Saint-Sulpice 75278 PARIS CEDEX 061983 


\section{PIERRE-ETIENNE HEYMANN \\ A PROPOS DE LA MISE EN SCENE DE MACBETH \\ PAR «LA ROSE DES VENTS» \\ A VILLENEUVE D'ASCQ}

\section{Le texte}

Le travail se fait à partir d'une traduction de JeanMarie Lhote, qui manifeste un souci de littéralité et respecte autant que possible la métrique et la syntaxe de Shakespeare. Il s'agit d'un montage et non d'une version intégrale; il n'y a pas là de lèse-majesté, car le texte même de Macbeth ressemble à un montage cinématographique : on peut jouer sur les fondus-enchaînés, les interrogations sans réponses, les apparentes incohérences (scènes «manquantes») qui créent le suspense. Certains éléments seront accentués, par exemple la contraction du temps un des modes de fonctionnement de la tragédie élisabéthaine -- dans la succession des arrivées, de la lettre de Macbeth; du messager, de Macbeth lui-même. Des coupures opérées dans les dialogues informatifs, la suppression des redites (récits du supplice de Cawdor), ont pour but de renforcer la péripétie, mais aussi l'irruption de l'irrationnel dans l'univers de Macbeth et de Banquo. Pour des raisons qui ne sont pas seulement économiques, les personnages anonymes, «porteurs de récits», (soldat, (I.ii) serviteurs, médecins, nobles) sont rassemblés en un seul personnage, porteur de mort. Les coupures n'excèdent pas dix pour cent du texte, ce qui donne une représentation de deux heures et demie. Aucun passage n'a été supprimé dans le texte des protagonistes, mais on a réduit la scène entre Malcolm et Macduff, qui correspond à une problématique à laquelle le public du XXe siècle est étranger. La scène iv de l'acte IV a été ramenée à cent vingt-six vers. Mais le tissu métaphorique n'a fait l'objet d'aucune réduction, la rhétorique n'a subi aucune mise à plat.

La langue

La première scène des sorcières (sauf le vers-clé de la 
pièce) ainsi que le début de la scène du chaudron et la scène du portier se joueront en anglais. Le portier parle un mélange d'anglais et de français (le double-jeu étant un des thèmes de la pièce). Garder l'anglais dans cette scène permet aussi de pallier la difficulté de traduction ( $«$ knock, knock ...»). En outre, le portier ne trouve pas ses clés, ce qui justifie la prolongation des coups.

\section{Les personnages}

Malcolm est traité comme le «gamin» décrit par Macbeth, il est vu à travers le prisme déformant de sa vision. Sa scène avec Macduff est intégrée dans la thématique de la double vérité (le vrai Malcolm est-il celui du début ou de la fin de la scène ?)

Les enfants: Fleance, le fils de Macduff, et l'enfantsorcière sont joués par un seul et même enfant pour des raisons dramaturgiques : dans les scènes avec Banquo, Fleance est toujours en scène, donnant à Macbeth l'image obsédante d'une paternité heureuse.

Le messager : Macbeth le traite de «face blême», c'est-à-dire, au sens figuré, «blanc de peur», mais aussi, au sens propre, personnage enfariné, bouffon, ce qui rejoint l'élément que Faulkner a pris à Shakespeare : l'idiot (ici le soldat blessé de la deuxième scène), amnésique et témoin de toute l'histoire, personnage comparable au «Mongol», ou «médecin des âmes», qui apparaît dans les rêves des moribonds.

\section{Le décor}

Il est inspiré par le thème de la stérilité du couple Macbeth, dont le seul objet est la recherche du pouvoir politique (le meurtre de Duncan étant conçu comme le serait l'enfant absent). Le décor, le lieu stérile, c'est le théâtre lui-même, cube de béton fonctionnel, effondré, envahi par les ronces et les scories, matériaux inverses de celui de la forêt de Birnam. 
La forêt de Birnam sera un plafond qui descendra au ras de la tête des spectateurs, assurant ainsi l'effet de rétrécissement de l'espace vital de Macbeth. L'échec relatif des représentations de Macbeth tient à la confusion entre Macbeth et les pièces historiques (trompettes, étendards, armées), alors qu'il s'agit d'une tragédie intimiste, où il faut jouer à la fois sur le proche (monologues très près du spectateur, parfois chuchotés) et le lointain (profondeur de champ pour les images obsessionnelles). Cette recherche et celle qui se rapporte au thème de la stérilité ont déterminé la scénographie. La réduction de l'espace du spectateur en même temps que de celui de Macbeth répond au projet d'un théâtre cathartique, qui provoque l'identification du spectateur avec Macbeth; car tout le monde est porteur de Macbeth (même Banquo n'est pas un héros positif) et la différence est due au hasard. L'espace sera complètement «baroque»: le public disposé en angle, avec focalisation de deux lieux opposés (lieu du «non-dit»; lieu du «dit», du discours) que recoupe le cercle de jeu des sorciers.

\section{Le surnaturel}

La direction du travail consiste, en radicalisant les incohérences, à essayer de traiter la pièce comme le rêve $\mathrm{du}$ personnage principal, car le caractère onirique de Macbeth représente un aspect fondamental de sa modernité.Le spectre sera sur la scène, puisque Shakespeare le veut ainsi. Les sorcières s'incarneront dans une famille de saltimbanques : un prestidigitateur, une femme et un enfant. L'actrice accomplit un travail de profération lyrique apparenté aux recherches musicales contemporaines. La prédiction s'adresse à Macbeth sous la forme d'un tour du prestidigitateur, qui fait sortir de son corps des cartes, dont la troisième est un roi. Le seul élément de mystère réside dans la question : comment les sorcières savent-elles les noms de Macbeth et de Banquo?

La deuxième rencontre avec les sorcières mettra en scène un chariot, où les apparitions seront le fruit de la 
magie de l'acteur (passage de l'illusion de la manipulation à celle du théâtre). La comédienne en qui s'incarnent les esprits jouera une scène de transe comparable à celles décrites par Michel Leyris dans ses récits de la possession en Ethiopie, ou aux scènes d'hystérie observées à la Salpêtrière.

Cette mise en scène de Macbeth sera un spectacle très simple, insistant sur l'élément ludique du théâtre shakespearien. Le travail des acteurs fait la part du hasard des trouvailles au cours des répétitions (rôle de Fleance, mise en scène $d u$ banquet). Pour faire cuvre d'art, il faut recréer chaque fois le code, parfois en rencontrant à nouveau des codes esthétiques archaïques. 